\title{
MicroRNA-650 targets inhibitor of growth 4 to promote colorectal cancer progression via mitogen activated protein kinase signaling
}

\author{
QI YOU ${ }^{1,2^{*}}$, HUINING LI $^{1,3^{*}}$, YAO LIU $^{4 *}$, YANGYANG XU $^{5}$, SUSHENG MIAO ${ }^{4}$, GUODONG YAO 6 , \\ YINGWEI XUE ${ }^{2}$, JINGSHU GENG ${ }^{6}$, XIAOMING JIN ${ }^{1}$ and HONGXUE MENG ${ }^{1,6}$ \\ ${ }^{1}$ Department of Pathology, Basic Research College, Harbin Medical University; ${ }^{2}$ Department of \\ Gastroenterology, Harbin Medical University Cancer Hospital; ${ }^{3}$ Department of Pathology, The First Affiliated \\ Hospital of Heilongjiang University of Chinese Medicine; Departments of ${ }^{4}$ Otolaryngology, ${ }^{5}$ Urology and \\ ${ }^{6}$ Pathology, Harbin Medical University Cancer Hospital, Harbin, Heilongjiang 150081, P.R. China
}

Received April 4, 2017; Accepted November 29, 2017

DOI: $10.3892 / \mathrm{ol} .2018 .8910$

\begin{abstract}
Colorectal cancer (CRC) is the third most common malignant disease globally and causes numerous cancer-associated mortalities; however, the underlying molecular mechanisms remain unresolved. MicroRNAs (miRs) are endogenous noncoding RNAs that regulate post-transcriptional gene silencing by annealing to partially complementary sequences in the 3'-untranslated regions of target mRNAs. In the present study, expression of the tumor suppressor gene inhibitor of growth protein 4 (ING4) in cell lines was investigated using reverse transcription-quantitative polymerase chain reaction and western blotting. miR-650 overexpression promoted CRC cell proliferation and migration by targeting ING4 when the cells were transfected with the miR-650 mimics. Additionally, overexpression of miR-650 increased the epithelial-mesenchymal transition and activation of the Ras homolog gene family member A/Ras-related C3 botulinum toxin GTPase. Extracellular signal-regulated kinases and p38 mitogen-activated protein kinase signaling were markedly activated when miR-650 was increased in CRC cells. Combined, the results
\end{abstract}

Correspondence to: Professor Xiaoming Jin, Department of Pathology, Basic Research College, Harbin Medical University, 169 Xuefu Road, Harbin, Heilongjiang 150081, P.R. China E-mail: jinxm55@163.com

Professor Hongxue Meng, Department of Pathology, Harbin Medical University Cancer Hospital, 169 Xuefu Road, Harbin, Heilongjiang 150081, P.R. China

E-mail: 13904649820@163.com

*Contributed equally

Key words: colorectal cancer, inhibitor of growth 4, mitogen activated protein kinase signaling, micro RNA-650, tumor progression indicate the mechanism underlying the miR-650 promotion of $\mathrm{CRC}$ progression, and provide promising potential biomarkers for the prognosis and treatment of CRC.

\section{Introduction}

Colorectal cancer (CRC), a cancer that arises from uncontrolled cell growth in the colon, rectum or the appendix, has become the third most common malignant disease worldwide with a yearly increasing incidence and mortality rate, and it is among the leading causes of cancer-associated mortality (1). It is the fourth leading cause of cancer-associated mortality in China (2). The incidence of CRC is increasing rapidly with $\sim 1$ million new CRC cases reported annually (3). In developed countries, the mortality of CRC increased to $33 \%$ between 2010 to 2013 (3). Although the 5-year survival rate of patients with $\mathrm{CRC}$ has been improved from $22-47 \%$ in the last 30 years with advancements in early diagnosis and therapeutic interventions, the overall survival rate remains pessimistic (3). The pathogenesis of CRC is not yet completely understood. It is currently known that colorectal carcinogenesis involves numerous molecular processes, including the activation of oncogenes, the mutation of mismatch repair genes or the inactivation of tumor suppressor genes, which affect the proliferation, migration, invasion and apoptosis of cancer cells, among other features (2).

Inhibitor of growth protein 4 (ING4), encoded by the ING4 gene, serves an essential function in cancer-associated cellular progression. It can interact with p53 and act as a tumor suppressor, thus affecting cell proliferation, migration and apoptosis, angiogenesis, contact inhibition and the DNA damage response $(4,5)$. Previous studies have demonstrated that ING4 expression is decreased in various types of cancer, including lung cancer, gastric carcinoma, colon cancer, breast cancer, melanoma and hepatocellular carcinoma, suggesting that the function of ING4 is to suppress tumor growth, angiogenesis and invasion in a number of types of cancer (6). Furthermore, overexpression of ING4 has been demonstrated to impair colony-forming efficiency, reduce the population of cells in the $S$ phase and to induce cancer cell migration, invasion and apoptosis $(7,8)$. In a 
previous study, it was identified that ING4 protein expression was downregulated in adenoma relative to in the normal mucosa, and was further decreased in CRC tissues (9). Furthermore, the suppression of ING4 expression was also associated with a more advanced Dukes stage (9). Therefore, it was hypothesized that ING4 serves important functions in colorectal carcinoma progression; however, the underlying molecular mechanism remains unresolved.

MicroRNAs (miR) are small noncoding RNA strands of 19-25 nucleotides in length. MiRs anneal inexactly to complementary sequences in the 3'-untranslated region (3'-UTR) of the target mRNAs of protein-coding genes and trigger cleavage of these target mRNAs, or they inhibit protein translation as key post-transcriptional regulators (10). In addition to gene inactivation, increasing evidence has identified that miRs are involved in a number of biological processes, including cell proliferation, differentiation, metastasis, apoptosis and immune responses (11). Furthermore, miRs may function as tumor suppressors or oncogenes in tumorigenesis, and have demonstrated prognostic significance for several tumor types $(12,13)$. miR-650 is a previously reported miR, which has been revealed to target ING4 in order to promote gastric cancer tumorigenicity (14). Further investigation indicates that miR-650 targets the promoter region of the NDRG2 gene and represses its transcription (15). The upregulation of miR-650 has been associated with ING4 downregulation and the progression of hepatocellular carcinoma (16). In an additional study, miR-650 was reported to be upregulated in several types of human colorectal cancer (17). However, the underlying molecular mechanism of miR-650 in CRC progression remains unclear.

Mitogen-activated protein kinases (MAPKs) belong to a family of serine/threonine-specific protein kinases, including three major MAPKs: Extracellular signal-regulated kinases (ERK1/2), c-Jun N-terminal kinases and p38 MAPKs $(18,19)$. MAPKs are involved in directing cellular responses to a diverse array of stimuli, including mitogens, osmotic stress, heat shock proteins and proinflammatory cytokines (20). MAPKs also function as essential modulators in signal transduction pathways, regulating gene transcription in response to external stimuli $(18,21,22)$. Furthermore, MAPKs have been identified to serve important functions in mediating cell proliferation, differentiation, transformation and apoptosis, and are involved in the development and progression of tumors (23).

In the present study, it was demonstrated that ING4 was a target gene of miR-650 in CRC. Elevated levels of miR-650 following transfection with miR-650 mimics contributed to increased cell vitality, elevated cell invasion and epithelial-to-mesenchymal transition (EMT). Overexpression of miR-650 induced the activation of Ras homolog gene family member A (RhoA)/Ras-related C3 botulinum toxin (Rac1) GTPase and MAPK signaling. These results indicate that miR-650 serves an important function in promoting CRC progression, and suggest that miR-650 and ING4 may be promising biomarkers for $\mathrm{CRC}$ diagnosis and therapy.

\section{Materials and methods}

Cell lines. Cell lines derived from human CRC (SW480, SW620, RKO, 320DM, 320HSR, NCI-H716, H508) and the normal liver cell line CCD841CoN used in this study were purchased from the cell bank of the American Type Culture Collection (Manassas, VA, USA).

Reagents. MTT was purchased from Sigma-Aldrich (Merck KGaA, Darmstadt, Germany). Four synthetic, chemically modified short double-stranded RNA oligonucleotides (miR-650 mimics, mimics negative control, miR-650 inhibitor and inhibitor negative control) were obtained from Guangzhou RiboBio Co., Ltd. (Guangzhou, China). Primary antibodies against p38 (\#9212), phosphorylated (p)-p38 (\#4511), ERK1/2 (\#4695), p-ERK1/2 (\#4370) and RhoA (\#2117) were obtained from Cell Signaling Technology, Inc. (Danvers, MA, USA); active Rac1 (\#26903) and active RhoA (\#26904) from NewEast Biosciences (King of Prussia, PA, USA); E-cadherin (ab1416), $\beta$-catenin (ab32572), Rac1 (ab33186) and FITC-conjugated anti-rat (ab6717)/anti-mouse IgG (ab6785) secondary antibodies from Abcam (Cambridge, UK); $\alpha$-smooth muscle actin ( $\alpha$-SMA, 55135-1-AP) from ProteinTech Group, Inc. (Chicago, IL, USA); vimentin (BM0135) from Wuhan Boster Biological Technology, Ltd. (Wuhan, China); and $\beta$-actin (sc-58673) and HRP conjugated goat anti-rabbit/ goat anti-mouse secondary antibodies (sc-2004/sc-2005) from Santa Cruz Biotechnology, Inc. (Dallas, TX, USA). The primary antibody ING4 (\#40-7700) and the fluorescent dye Alexa Fluor 546 conjugated phalloidin (A22283, 1:5,000) were purchased from Invitrogen (Thermo Fisher Scientific, Inc., Waltham, MA, USA).

Cell culture. Cells were cultured in L15 medium (Biological Industries, Kibbutz Beit Haemek, Israel) supplemented with $10 \%$ fetal bovine serum (Biological Industries USA, Cromwell, CT, USA), $100 \mathrm{U} / \mathrm{ml}$ penicillin and $100 \mu \mathrm{g} / \mathrm{ml}$ streptomycin, at $37^{\circ} \mathrm{C}$ in a humidified incubator containing $5 \% \mathrm{CO}_{2}$.

Transfection. For transfection, miR-650 mimics (100 nM), miR-650 inhibitors and their aforementioned negative controls were transfected into the SW620 and SW480 cells using Lipofectamine ${ }^{\circledR} 2000$ (Invitrogen; Thermo Fisher Scientific, Inc.) according to the manufacturer's protocol. ING4 3'UTR and the mutant 3'UTR were inserted into the plasmid with a luciferase reporter gene and then ING4 3'UTR and the mutant 3'UTR were transfected into the HEK293T cells. The sequences of the primers are listed as follows: ING4-3'F-UTR-P1: 5'-GCGCGCAAGCTTCAA CACAGTTTCTTCCACATCCCC-3' m-ING4-3'R-UTR-P1: 5'-GCGCTCTAGACTCTACAAAACATTCTTCCATTGTAT AGCTTTTATTTAC-3'. ING4-3' F-UTR-P4: 5'-GCGCAT TCTAGACTCTACAATAAACACAGCAGGC-3' m-ING43'R-UTR-P4: 5'-GCGCGCTCTAGACTCTACAATAAACAT TCTTTCCCATCTTGTATAGCTTTTATTTACCTACCC-3'.

For ING4 knockdown, the specific shING4, sense: 5'-GATCCG AGGCTGATCTCAAGGAGAAATTCAAGAGATTTCTCCT TGAGATCAGCCTCAGA-3'; negative control short hairpin RNA (shRNA), sense: 5'-AAGCTGAAGTACAACCTTCTT CAAGAGAGAAGGTTGTACTTCAGCTTAG-3' was transfected into SW480 and SW620 cells using Lipofectamine 2000 (Thermo Fisher Scientific, Inc.) with standard transfection procedures. At $48 \mathrm{~h}$ post transfection, $600 \mu \mathrm{g} / \mathrm{ml} \mathrm{G} 418$ (Sigma-Adrich; Merck KGaA) was added to select stable transfectants, and individual clones were isolated and maintained in 
a medium containing G418 (200 $\mu \mathrm{g} / \mathrm{ml})$. For ING4 overexpression, ING4 was inserted into the pcDNA3.1 vector (Invitrogen; Thermo Fisher Scientific, Inc.), which was then transfected into the cells. Transfection was performed using Lipofectamine 2000 (Thermo Fisher Scientific, Inc.) according to the manufacturer's protocol. At $48 \mathrm{~h}$ post transfection, $600 \mu \mathrm{g} / \mathrm{ml} \mathrm{G} 418$ (Sigma-Aldrich; Thermo Fisher Scientific, Inc.) was added to select stable transfectants, and individual clones were isolated and maintained in a medium containing G418 $(200 \mu \mathrm{g} / \mathrm{ml})$.

Cell viability assay. An MTT assay was used to determine cell viability. Individual wells of a 96-well plate were inoculated with $100 \mu 1$ of L15 medium containing $5 \times 10^{4}$ cells. Cells were transfected with miR-650 mimics, miR-650 inhibitors and their negative controls. A total of $20 \mu \mathrm{l}$ MTT solution $(0.5 \mathrm{mg} / \mathrm{ml})$ was added to each well and incubated at $37^{\circ} \mathrm{C}$ for $4 \mathrm{~h}$. The culture medium was removed and $200 \mu 1$ dimethyl sulfoxide was added to each well to dissolve the purple formazan for $10 \mathrm{~min}$ at room temperature. The absorbance values were read at $570 \mathrm{~nm}$ using an Infinite m200pro microplate spectrophotometer (Tecan Group, Ltd., Männedorf, Switzerland).

Wound healing assay. Cells were seeded in a 24-well plate and cultured to $100 \%$ confluency, following which the cell monolayer was scratched in a straight line using a pipette tip $(200 \mu 1)$. In order to remove the debris and smooth the edge of the scratch, culture medium was removed and the wells were washed three times with $1 \mathrm{ml}$ growth medium. After $24 \mathrm{~h}$ of culture, the scratch was viewed using a microscope (24).

Total RNA extraction and reverse transcription-quantitative polymerase chain reaction (RT-qPCR) analysis. Total RNA from the cultured cell lines was isolated using TRIzol ${ }^{\circledR}$ reagent (Life Sciences; Thermo Fisher Scientific, Inc.) according to the manufacturer's protocol. The relative expression of miR-650 and ING4 were determined using the SYBR Green I method on a CFX96 Real-Time C1000 Touch Thermocycler system (Bio-Rad Laboratories, Inc., Hercules, CA, USA) with U6 and $G A P D H$ as the internal controls. Each reaction was carried out in triplicate.

Immunofluorescence staining. Cells cultured in 6-well plates were fixed with $4 \%$ paraformaldehyde in PBS $(\mathrm{pH}=7.35)$ for $15 \mathrm{~min}$ at room temperature. Nonspecific binding was blocked for $2 \mathrm{~h}$ with goat serum (Sigma-Aldrich; Merck KGaA,), and the cells were subsequently incubated with the aforementioned primary antibodies against E-cadherin, $\beta$-catenin, $\alpha$-SMA and vimentin (all diluted to $1: 50$ ) at $4^{\circ} \mathrm{C}$ overnight. The cells were washed and incubated with the FITC-conjugated anti-rat (dilution, 1:2,000) or anti-mouse $\operatorname{IgG}$ (dilution, 1:2,000) secondary antibodies for $1 \mathrm{~h}$. The cells were visualized using a confocal microscope (magnification x100).

Protein extraction and western blotting. Cell samples were lysed in radioimmunoprecipitation assay buffer (Thermo Fisher Scientific, Inc.) with a protease inhibitor and phosphatase inhibitor cocktail (cat no. ab201119; Abcam), prior to protein extraction. Proteins were quantified using a Bio-Rad protein assay kit (cat no. 5000002, Bio-Rad Laboratories, Inc., Hercules, CA, USA), and equal quantities (10 $\mu \mathrm{g})$ were
A

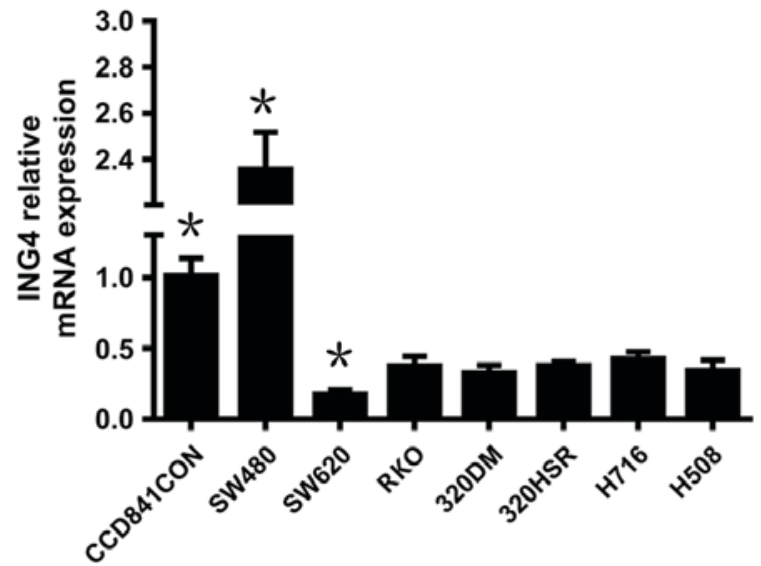

B

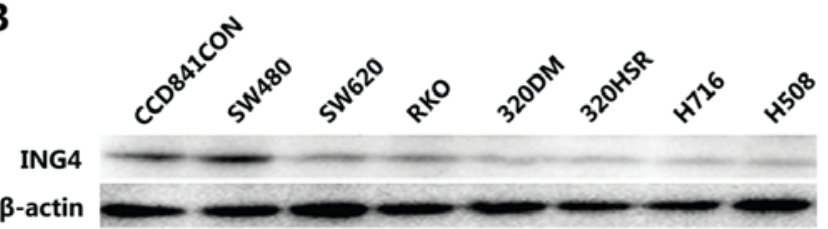

Figure 1. Expression of ING4 in various CRC cell lines. (A) The mRNA levels of ING4 in various CRC cell lines, and in the normal colonic epithelial cell line CCD841CoN, were detected using reverse transcription-quantitative polymerase chain reaction and then normalized against $\beta$-actin. (B) The protein level of ING4 in various CRC cell lines and the normal colonic epithelial cell line CCD841CoN was detected using western blotting. " $\mathrm{P}<0.05$ vs. all other CRC cell lines. $\beta$-actin was used as the internal control. CRC, colorectal cancer; miR, micro RNA; ING4, inhibitor of growth protein 4.

fractionated using SDS-PAGE (4\%) and transferred to a polyvinylidene fluoride membrane (EMD Millipore, Billerica, MA, USA). The membranes were blocked with 5\% bovine serum albumin (Sigma-Aldrich, Merck KGaA) for $2 \mathrm{~h}$ at room temperature with agitation, and then incubated with the aforementioned primary antibodies, including those against ING4, E-cadherin, $\beta$-catenin, $\alpha$-SMA, vimentin, RhoA, active RhoA, Rac1, active Rac1, ERK1/2, p-ERK1/2, p38 and p-p38, each at a dilution of 1:1,000, at $4^{\circ} \mathrm{C}$ overnight. Following three washes with Tris-buffered saline containing $0.5 \%$ Tween 20 , the membranes were incubated with horseradish peroxidase-conjugated secondary antibodies (dilution, 1:5,000) at room temperature for $1 \mathrm{~h}$. Bands were observed using an Odyssey infrared imaging system (LI-COR Biosciences, Lincoln, NE, USA). $\beta$-actin was used as the internal control.

Statistical analysis. All data are presented as the mean \pm standard error of the mean. Multiple comparisons were analyzed statistically using Mann-Whitney U test or one-way analysis of variance followed by a Student-Newman-Keuls post-hoc test. All analysis was performed using GraphPad Prism software version 5.0 (GraphPad Software, Inc., La Jolla, CA, USA). A two-tailed value of $\mathrm{P}<0.05$ was considered to indicate a statistically significant difference.

\section{Results}

The expression of ING4 in various $C R C$ cell lines. In order to investigate ING4 expression in CRC cells, including the cell lines SW480, SW620, RKO, 320DM, 320HSR, NCI-H716 and 
A

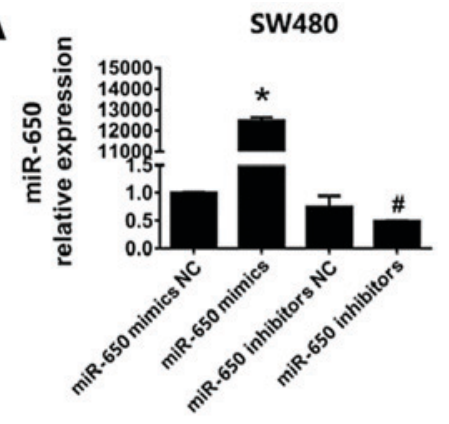

SW480

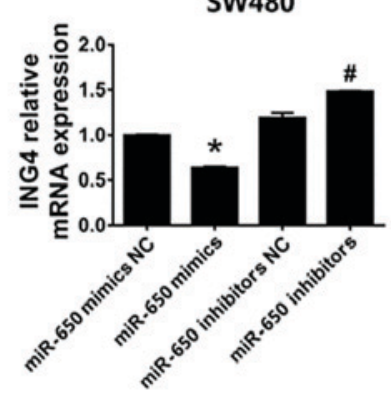

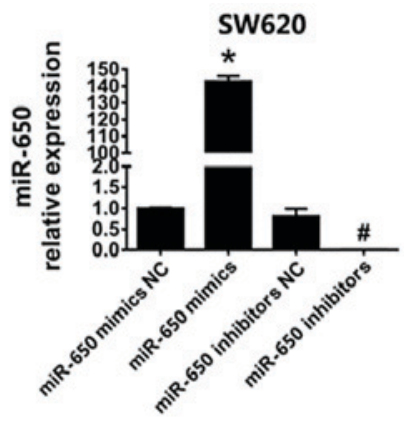

SW620

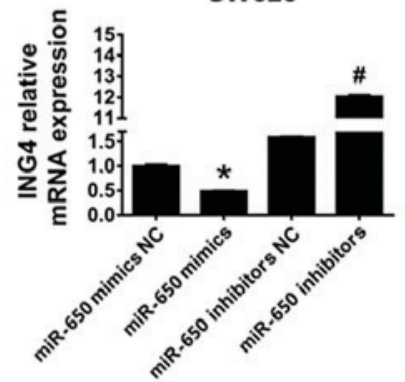

B
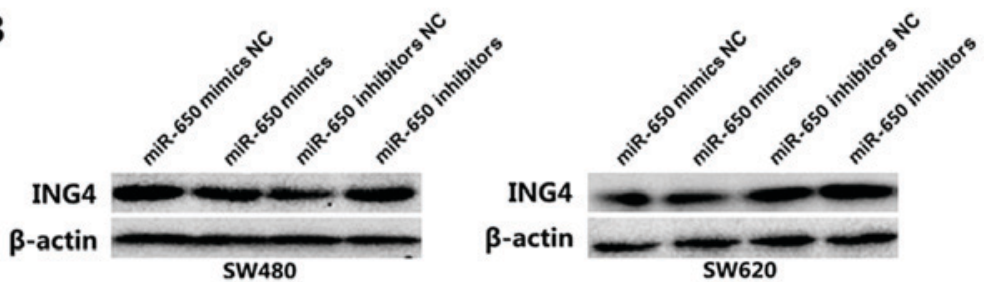

C
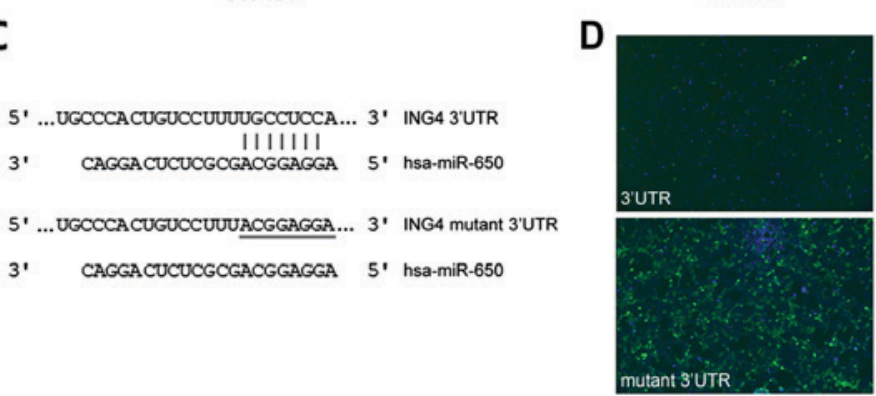

Figure 2. MiR-650 targets the 3'UTR of ING4 in CRC cells. (A) Reverse transcription-quantitative polymerase chain reaction analysis of the expression of miR-650 and ING4 in SW480 and SW620 cells following transfection with miR-650 mimics, miR-650 mimics NC, miR-650 inhibitors and miR-650 inhibitors NC. The relative expression of miR-650 and ING4 was normalized to that of $U 6$ and $\beta$-actin, respectively. (B) Western blot analysis of the protein levels of ING4 following miR-650 mimics transfection. (C) The core nucleotide sequence of ING4 3'UTR containing the predicted binding site matched by miR- 650 . The matched nucleotides are indicated with vertical lines, and the mutant residues in ING4 3'UTR are underlined. (D) Immunofluorescence verification the targeting of miR-650 to the 3'UTR of ING4. The ING4 3'UTR and its mutant were constructed into the plasmid with luciferase reporter and transfected with miR-650 mimics into HEK293T cells, respectively. Slides were analyzed under a light microscope (BX53; Olympus Corporation, Tokyo, Japan) using a BX3-URA fluorescence system (Olympus Corporation) (magnification $\mathrm{x} 100$ ). ${ }^{*} \mathrm{P}<0.05$ vs. control group.miR-650 mimics NC, miR-650 inhibitors and miR-650 inhibitors NC groups. ${ }^{.} \mathrm{P}<0.05$ vs.control group. miR-650 mimics NC, miR-650 mimics and miR-650 inhibitors NC groups. NC, negative control; miR, micro RNA; ING4, inhibitor of growth protein 4.

H508, RT-qPCR and western blotting were performed. The normal colonic epithelial cell line CCD841CoN was used as the control. As presented in Fig. 1A, the mRNA expression of ING4 was decreased compared with the control in all the CRC cell lines except SW480 $(\mathrm{P}<0.05)$, suggesting the decreasing trend of ING4 in CRC, which is consistent with the expression in tissues of patients (9). The expression level of ING4 in SW480 was markedly increased compared with the control $(\mathrm{P}<0.05)$. ING4 expression in SW620 was lower than all other CRC cell lines $(\mathrm{P}<0.05)$. Therefore, SW480 and SW620 cell lines were selected for subsequent experiments. The protein levels of ING4 in various cell lines revealed similar trends with the mRNA levels (Fig. 1B).
MiR-650 targets the 3'UTR of ING4 in CRC cells. It has been reported that miR-650 targets ING4 to promote gastric cancer tumorigenicity (14). Therefore, the present study investigated whether miR-650 targets ING4 in CRC. Following the transfection of miR-650 mimics, miR-650 mimics negative control (NC), miR-650 inhibitors and miR-650 inhibitors $\mathrm{NC}$, the level of miR-650, and the mRNA and protein levels of ING4 were analyzed using RT-qPCR and western blotting, respectively. As presented in Fig. 2A, the levels of miR-650 in SW480 and SW620 were significantly increased compared with those in the miR-650 mimics NC, miR-650 inhibitors and miR-650 inhibitors $\mathrm{NC}$ groups $(\mathrm{P}<0.05)$. As predicted, the mRNA level of $I N G 4$ was significantly attenuated following 

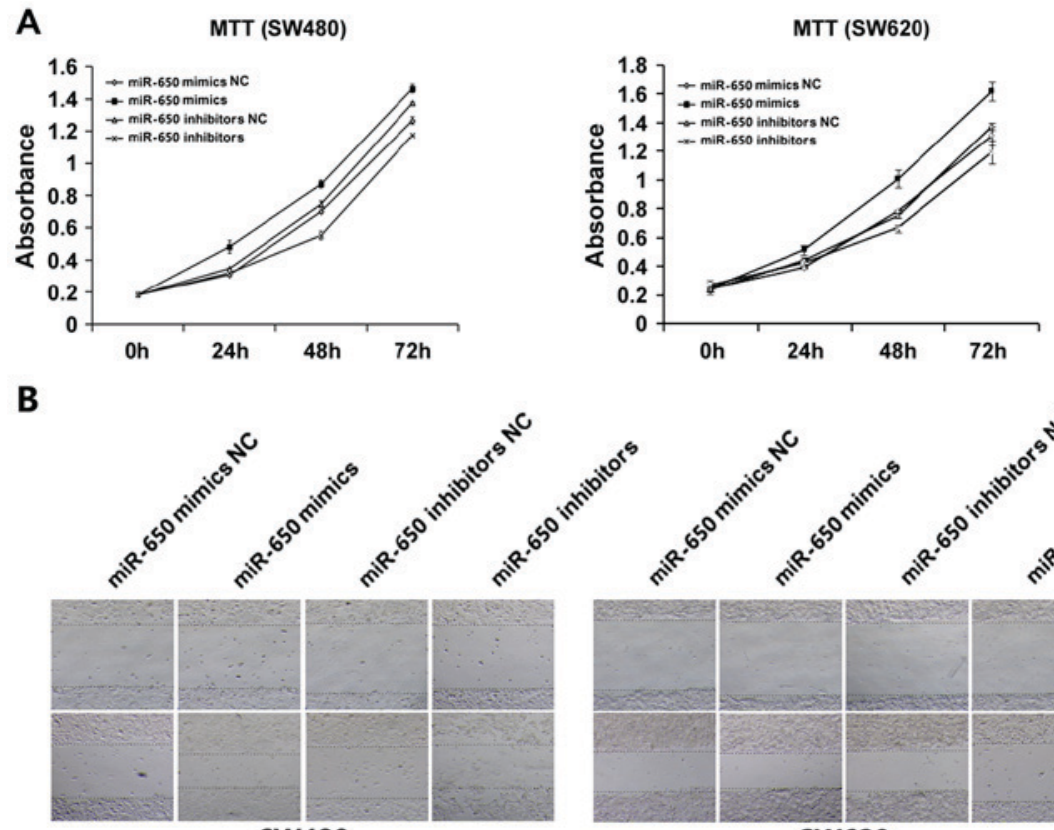

SW480

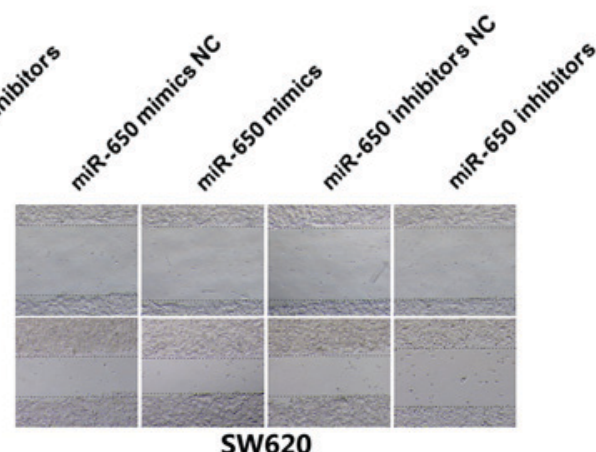

SW620

Figure 3. MiR-650 promotes the proliferation and migration of colorectal cancer cells. (A) An MTT assay was performed to examine the cell proliferation in SW480 and SW620 cells following transfection with miR-650 mimics, miR-650 mimics NC, miR-650 inhibitors and miR-650 inhibitors NC. (B) Scratch test for the migration of SW480 and SW620 cells following transfection. NC, negative control; miR, micro RNA.

miR-650 transfection, relative to the controls $(\mathrm{P}<0.05)$ The changes in ING4 protein levels were consistent with the mRNA levels (Fig. 2B).

According to the miRDB and TargetScan databases, miR-650 was predicted to bind to the 3'UTR of ING4, and the core sequence containing the binding site is presented in Fig. 2C. The ING4 3'UTR and the mutant 3'UTR were inserted into the plasmid with a luciferase reporter gene. Following the co-transfection of miR-650 mimics with the plasmid containing ING4 3'UTR, almost no fluorescence was observed. However, when co-transfected with the mutant 3'UTR, marked fluorescence was observed (Fig. 2D). These results indicate that miR-650 negatively regulates the expression of ING4 by targeting the 3'UTR of ING4 transcripts in CRC cells.

MiR-650 promotes the proliferation and migration of CRC cells. To further resolve the function of miR-650 during tumorigenesis, SW480 and SW620 cells were transfected with miR-650 mimics, miR-650 mimics NC, miR-650 inhibitors and miR-650 inhibitors NC. An MTT assay was performed to examine cell viability. The results revealed that cell proliferation in the group transfected with miR-650 mimics was increased compared with in the group transfected with miR-650 mimics NC; proliferation in the group transfected with the miR-650 inhibitor was decreased compared with in the group transfected with the miR-650 inhibitor NC (Fig. 3A).

The effect of miR-650 on the cell migration was evaluated using a wound healing assay. The results indicated that transfection of miR-650 mimics increased the migration of SW480 cell lines compared with the respective control groups (Fig. 3B), which suggest that miR-650 upregulation may be associated with tumor growth and migration in CRC.
MiR-650 induces EMT in SW620 and SW480 cell lines. Following transfection with miR-650 mimics, various morphological changes, including spindle-shaped and fibroblastic cell morphology, as well as scattering and decreased cell-cell contact were observed in SW480 and SW620 cells (Fig. 4A), suggesting that these cells may undergo EMT progression. EMT is an essential event in tumor invasion and metastasis, which is characterized by the decreased presence of certain epithelial markers (E-cadherin and $\beta$-catenin) and upregulated mesenchymal markers ( $\alpha$-SMA and vimentin) (25). In the present study, western blotting was used to further verify this EMT progression in the cell lines. As presented in Fig. 4B, in miR-650 mimics-transfected cells, the expression of E-cadherin and $\beta$-catenin was markedly decreased. On the contrary, mesenchymal markers, including vimentin and $\alpha$-SMA, were markedly elevated in SW480 and SW620 cells (Fig. 4B). Immunofluorescence indicated decreased expression of E-cadherin, $\beta$-catenin and increased expression of SMA in miR-650 mimics-transfected cells compared with control (Fig. 4C). These data reinforce that the overexpression of miR-650 induces EMT in SW480 and SW620 cell lines.

MiR-650 induces the activation of Rho/Rac GTPases in SW620 and SW480 cell lines. Since miR-650 transfected cells exhibited morphological alterations, the effects of miR-650 on modulating cytoskeleton rearrangement were further investigated. F-actin staining by phalloidin revealed that stress fibers and lamellipodia were observed in miR-650 transfected cells, but fewer were present in control cells. By contrast, miR-650 inhibitor group exhibited reduced stress fibers in the SW620 and SW480 cells (Fig. 5A). During tumor progression, activation of Rho-GTPase contributes to actin cytoskeleton reorganization, which in turn causes disruption of adherent junctions $(26,27)$. Therefore, considering 


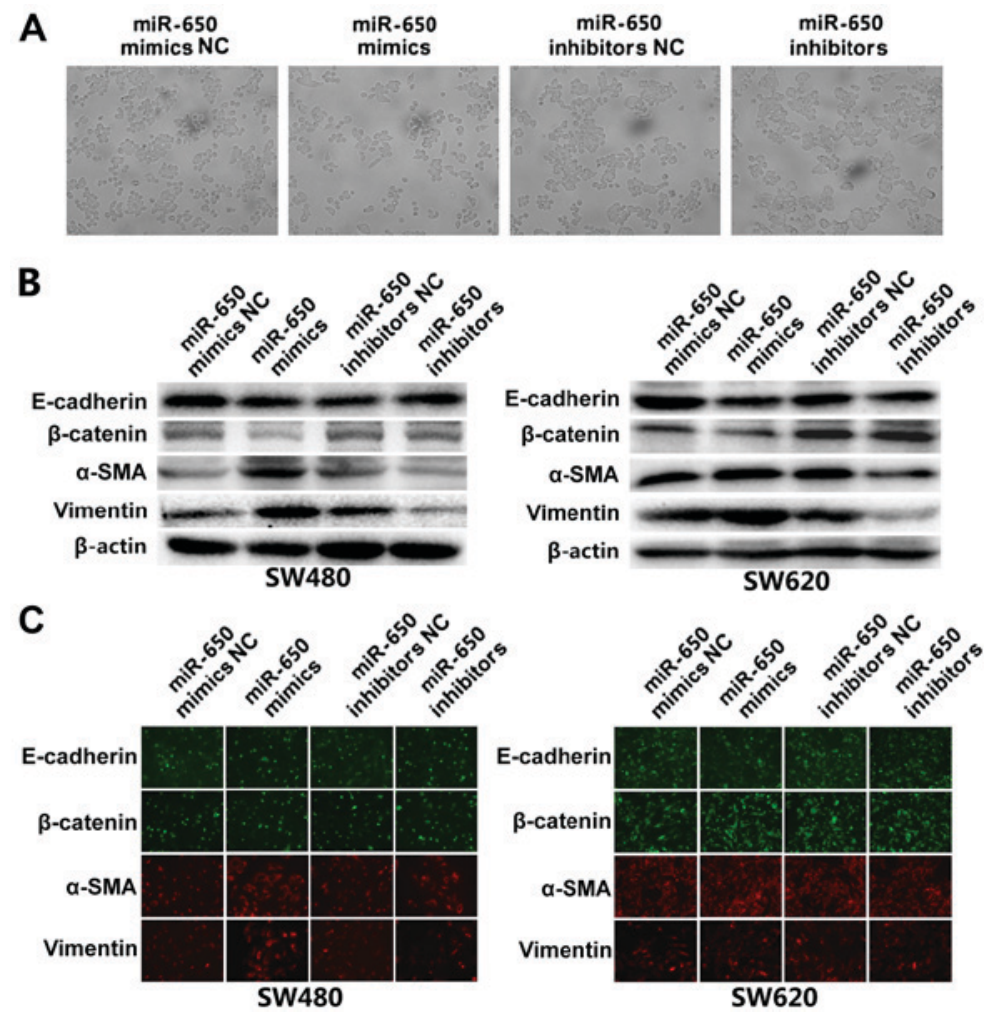

Figure 4. miR-650 promotes the EMT progression of colorectal cancer cells. (A) Cell morphology following miR-650 mimics transfection. The cells revealed a relative scattering and fibroblastic morphology. miR-650 mimics NC, miR-650 inhibitors and miR-650 inhibitors NC were used as the controls. (B) Western blot analysis of the expression of EMT markers in SW480 and SW620 cells following transfection, including E-cadherin, $\beta$-catenin, $\alpha$-SMA and vimentin. (C) Immunofluorescence for the expression of these markers. Slides were analyzed under a light microscope (BX53; Olympus Corporation, Tokyo, Japan) using a BX3-URA fluorescence system (Olympus) (magnification x100). MiR, micro RNA; EMT, epithelial mesenchymal transition; NC, negative control.

A

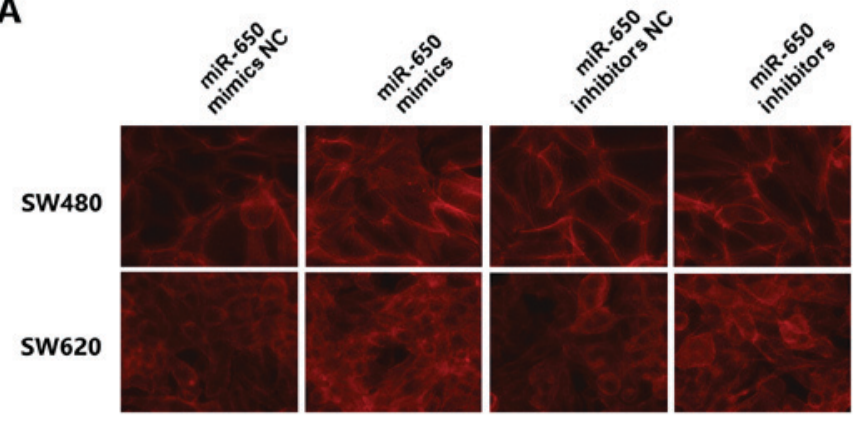

B

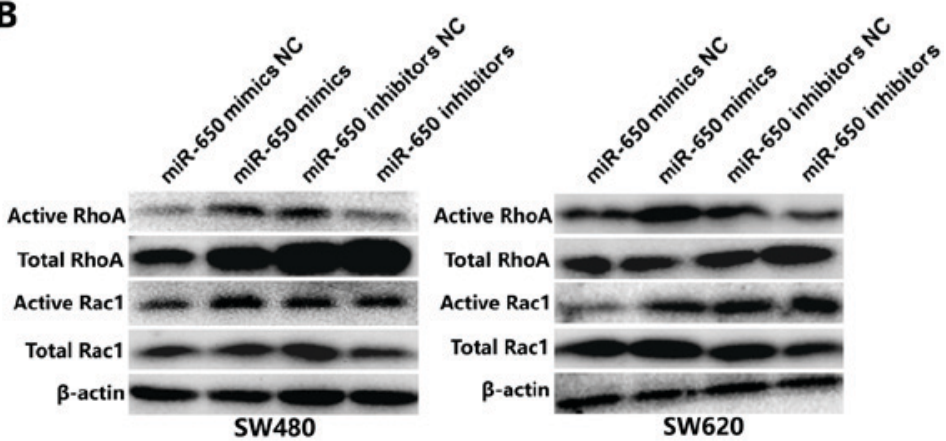

Figure 5. MiR-650 induces the activation of Rho/Rac GTPases in colorectal cancer cells. (A) F-actin staining by Alexa Fluor 546 conjugated Phalloidin. (B) Western blot analysis of the expression of RhoA, Rac1 and their active forms in SW480 and SW620 cells following transfection. MiR, micro RNA; RhoA, Ras homolog gene family member A; Rac1, Ras-related C3 botulinum toxin substrate 1 .

the morphological alterations, it was further determined whether Rho-GTPases were activated following miR-650 overexpression. Western blotting results revealed that the active form of RhoA was increased in SW480 and SW620 


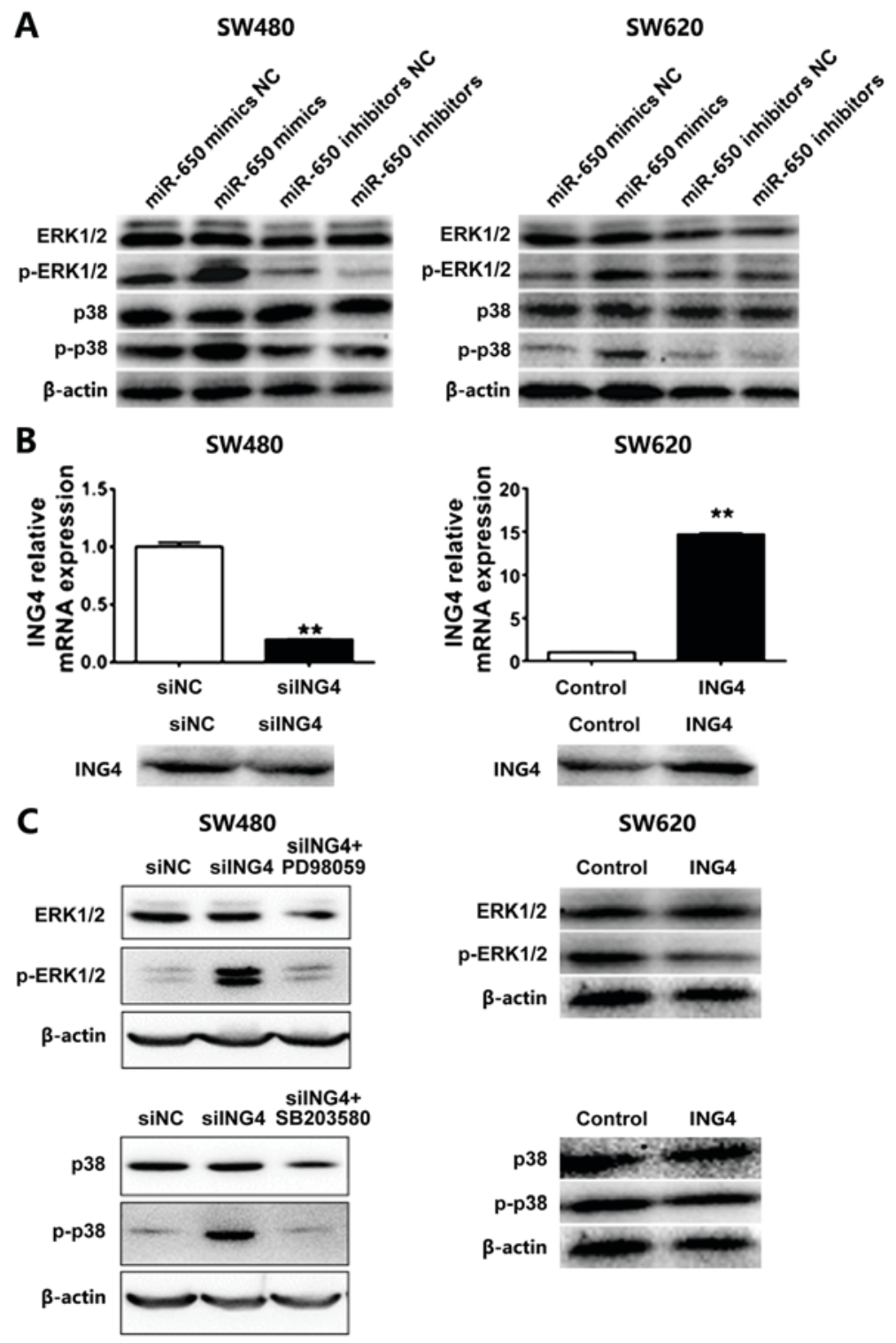

Figure 6. miR-650 exerts its function through the activation of ERK and p38 MAPK. (A) Western blot analysis of the expression of ERK and p38 MAPK in SW480 and SW620 cells following transfection with miR-650 mimics, miR-650 mimics NC, miR-650 inhibitors and miR-650 inhibitors NC. (B) Reverse transcription-quantitative polymerase chain reaction and western blot analysis of the expression of ING4 in SW480 and SW620 cells following ING4 knockdown by siRNA and overexpression. (C) Western blot analysis of the expression of ERK and p38 MAPK in SW480 and SW620 cells following ING4 knockdown and overexpression. PD98059 and SB203580 are the inhibitors of ERK1/2 and p38 MAPK respectively. ${ }^{*} \mathrm{P}<0.05$ vs. control group. MAPK, mitogen activated protein kinase; miR, micro RNA; ERK, extracellular signal-related kinases; ING4, inhibitor of growth protein 4; si, small interfering.

cells when miR-650 was overexpressed, and decreased following transfection with the miR-650 inhibitor. The expression of active Rac1 was also elevated by miR-650 overexpression (Fig. 5B). There was no notable reduction in the expression of active Rac1 in miR-650 inhibitors-transfected cells compared with the NC-transfected group. These results indicate that miR-650 activates small GTPase Rac1 and RhoA, disrupting adherent junctions between cells and promoting actin cytoskeleton reorganization.

MiR-650 exerts its function through MAPK signaling. MAPK signaling serves functions in mediating cell proliferation, differentiation, transformation and apoptosis, and is involved in the development and progression of tumors (19). Promotion of tumor progression by miR-650 via the two major MAPKs, including ERK1/2 and p38 MAPK was investigated. Western blotting revealed that transfection of miR-650 elevates the levels of p-ERK1/2 and p-p38 MAPK, while the inhibition of miR-650 attenuates their expression in SW480 and SW620 cells (Fig. 6A). These results suggest that ERK and p38 MAPK may be involved in the modulation of tumor development and progression in CRC. To determine the association between ING4 and these MAPKs, ING4 was overexpressed in SW480 and SW620, respectively (Fig. 6B). Western blotting revealed that the levels of phosphorylated ERK and p38 MAPK were increased when ING4 was knocked down but decreased when ING4 was overexpressed (Fig. 6C). The application of the inhibitors of ERK and p38 MAPK could reduce their phosphorylation levels. Taken together, these results suggest that miR-650 targets ING4 and then activates ERK and p38 MAPK to promote CRC progression. 


\section{Discussion}

Colorectal cancer (CRC) has become the third most common type of malignant disease worldwide, and is one of the leading causes of cancer-associated mortality. However, the pathogenesis of CRC is not completely understood. Therefore, it is important to resolve the underlying molecular mechanism of CRC progression and carry out effective treatments to prevent and cure the disease. Emerging studies have demonstrated that miRs serve crucial functions in the progression of CRC (28).

At present, limited reports have demonstrated that miR-650 is involved in cancer. Mraz et al (29) reported that miR-650 expression is associated with the prognosis of chronic lymphocytic leukemia and has influences on B-cell proliferation. A high level of miR-650 was identified to be a prognostic indicator for lymph node involvement and more aggressive clinical outcomes of lung adenocarcinoma (30). In CRC, little information is known about the function of miR-650, except for its involvement in regulating expression of the NDRG gene (15). In the present study, it was demonstrated that in CRC, miR-650 targets ING4 and has effects on promoting proliferation, migration and activation of ERK and p38 MAPK which provides further evidence of the involvement of miR-650 in cancer progression.

ING4 was initially identified to be involved in the regulation of glioma growth and angiogenesis and interacting with

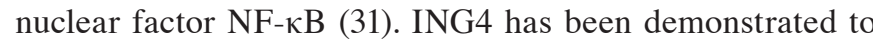
induce cell growth inhibition in human lung adenocarcinoma cells and gastric carcinoma cells $(32,33)$, inhibit melanoma cell invasion (34) and attenuate cellular transformation (35). Similarly, in the present study, inhibition of ING4 through the overexpression of miR-650 promoted CRC cell proliferation and migration (Fig. 3B).

In the present study, a series of assays were carried out to characterize the function of miR-650 in regulating CRC cell growth, invasiveness, EMT and actin cytoskeleton reorganization. The expression of ING4 in patient CRC tissues, compared with in the adjacent non-tumor tissues, was previously evaluated, and the results revealed that ING4 sharply decreased in cancer (36). To confirm the decreased level of ING4 in CRC, the expression of ING4 was evaluated in 8 CRC cell lines, which is consistent with the previous results. Two cell lines (SW480 and SW620) were selected for further experiments. miR-650 was overexpressed by transfecting miR-650 mimics into SW480 and SW620 cells, and inhibited by transfecting with an miR-650-inhibitor. The results demonstrated that overexpression of miR-650 significantly inhibited ING4. As ING4 is a tumor suppressor gene, the function of miR-650 in tumor progression was assessed, including cell growth, cell invasion, EMT and cell actin cytoskeleton reorganization. The underlying molecular mechanisms of miR-650 in promoting cancer progression were also evaluated. Western blotting results indicate that overexpression of miR-650 enhanced the activation of ERK and p38 MAPK. In addition, knockdown of ING4 results in elevated levels of p-ERK and p-p38 MAPK. These results suggest that miR-650 targets ING4 which functions upstream of ERK and p38 MAPK to promote CRC progression. Whether ING4 interacts with ERK and p38 MAPK or other cancer-associated proteins to activate ERK and p38 MAPK must still be further explored.

To the best of our knowledge, this is the first study to identify the critical functions of miR-650 in CRC. miR-650 may target ING4 to promote CRC progression through the MAPK signaling pathway. The present study not only evaluated the functions of miR-650 in CRC progression, but also provided a solid basis to explore the pathogenesis of and develop therapeutic strategies for CRC.

\section{Acknowledgements}

The authors would like to thank Professor Dawei Yuan (Geneis Beijing Co. Ltd., Beijing, China) for his technical assistance with cell culture support.

\section{Funding}

This work was supported by the National Nature Science Foundation of China (grant nos. 81600539, 81400443, 81372178), the Natural Science Foundation of Heilongjiang Province of China (grant nos. QC2012C041, LC2016038), the Foundation of Heilongjiang Administration of Traditional Chinese Medicine (Huining Li, grant no. ZHY16-032), the Chinese Postdoctoral Science Foundation (grant no. 2015M581472), the Special Financial Grant from the China Postdoctoral Science Foundation (grant no. 2016T90310), the Postdoctoral Science Foundation of Heilongjiang Province of China (grant nos. LBH-Z16101, LBH-TZ0616), the Heilongjiang Human Resources and Social Security Bureau (Hongxue Meng), the Harbin Special Fund Project for Science and Technology Innovation (grant no. 2016RAQXJ203) and the Youth Elite Training Foundation of Harbin Medical University Cancer Hospital (grant no. JY2016-06).

\section{Availability of data and materials}

All data that were generated or analyzed in this study are included in this manuscript.

\section{Author's contributions}

QY, HL and YL conceived and designed the study. YX and SM conducted the experiments. GY and YX performed the statistical analysis. JG, XJ and HM interpreted the statistical analysis, reviewed and final approved the version to be published. All authors read and approved the manuscript.

\section{Ethics approval and consent to participate}

Not applicable.

\section{Consent for publication}

Not applicable.

\section{Competing interests}

The authors declare that they have no competing interests. 


\section{References}

1. Chia VM, Newcomb PA, Bigler J, Morimoto LM, Thibodeau SN and Potter JD: Risk of microsatellite-unstable colorectal cancer is associated jointly with smoking and nonsteroidal anti-inflammatory drug use. Cancer Res 66: 6877-6883, 2006.

2. Zhou Y, Feng X, Liu YL, Ye SC, Wang H, Tan WK, Tian T, Qiu YM and Luo HS: Down-regulation of miR-126 is associated with colorectal cancer cells proliferation, migration and invasion by targeting IRS-1 via the AKT and ERK1/2 signaling pathways. PLoS One 8: e81203, 2013

3. Chen Z, He X, Xia W, Huang Q, Zhang Z, Ye J, Ni C, Wu P, Wu D, $\mathrm{Xu}$ J, et al: Prognostic value and clinicopathological differences of HIFs in colorectal cancer: Evidence from meta-analysis. PLoS One 8: e80337, 2013.

4. Guo Y, Meng X, Wang Q, Wang Y and Shang H: The ING4 binding with p53 and induced $\mathrm{p} 53$ acetylation were attenuated by human papillomavirus 16 E6. PLoS One 8: e71453, 2013.

5. Li J and Li G: Cell cycle regulator ING4 is a suppressor of melanoma angiogenesis that is regulated by the metastasis suppressor BRMS1. Cancer Res 70: 10445-10453, 2010.

6. Byron SA, Min E, Thal TS, Hostetter G, Watanabe AT, Azorsa DO, Little TH, Tapia C and Kim S: Negative regulation of NF- $\mathrm{KB}$ by the ING4 tumor suppressor in breast cancer. PLoS One 7: e46823, 2012

7. Culurgioni S, Muñoz IG, Moreno A, Palacios A, Villate M, Palmero I, Montoya G and Blanco FJ: Crystal structure of inhibitor of growth 4 (ING4) dimerization domain reveals functional organization of ING family of chromatin-binding proteins. J Biol Chem 287: 10876-10884, 2012.

8. Palacios A, Moreno A, Oliveira BL, Rivera T, Prieto J, Garcia P, Fernández-Fernández MR, Bernadó P, Palmero I and Blanco FJ: The dimeric structure and the bivalent recognition of $\mathrm{H} 3 \mathrm{~K} 4 \mathrm{me} 3$ by the tumor suppressor ING4 suggests a mechanism for enhanced targeting of the HBO1 complex to chromatin. J Mol Biol 396: 1117-1127, 2010.

9. You Q, Wang XS, Fu SB and Jin XM: Downregulated expression of inhibitor of growth 4 (ING4) in advanced colorectal cancers: A non-randomized experimental study. Pathol Oncol Res 17: 473-477, 2011

10. Huang WC, Chan SH, Jang TH, Chang JW, Ko YC, Yen TC, Chiang SL, Chiang WF, Shieh TY, Liao CT, et al: miRNA-491-5p and GIT1 serve as modulators and biomarkers for oral squamous cell carcinoma invasion and metastasis. Cancer Res 74: 751-764, 2014.

11. Esquela-Kerscher A and Slack FJ: Oncomirs-microRNAs with a role in cancer. Nat Rev Cancer 6: 259-269, 2006

12. Chen D, Chen Z, Jin Y, Dragas D, Zhang L, Adjei BS, Wang A, Dai Y and Zhou X: MicroRNA-99 family members suppress Homeobox A1 expression in epithelial cells. PLoS One 8: e80625, 2013.

13. Sugihara $H$, Ishimoto $T$, Watanabe $M$, Sawayama H, Iwatsuki M, Baba Y, Komohara Y, Takeya M and Baba H: Identification of miR-30 * regulation of Bmil expression mediated by tumor-associated macrophages in gastrointestinal cancer. PLoS One 8: e81839,2013.

14. Zhang X, Zhu W, Zhang J, Huo S, Zhou L, Gu Z and Zhang M: MicroRNA-650 targets ING4 to promote gastric cancer tumorigenicity. Biochem Bioph Res Commun 395: 275-280, 2010.

15. Feng L, Xie $\mathrm{Y}$, Zhang $\mathrm{H}$ and $\mathrm{Wu} \mathrm{Y:} \mathrm{Down-regulation} \mathrm{of}$ NDRG2 gene expression in human colorectal cancer involves promoter methylation and microRNA-650. Biochem Bioph Res Commun 406: 534-538, 2011.

16. Zeng ZL, Li FJ, Gao F, Sun DS and Yao L: Upregulation of miR-650 is correlated with down-regulation of ING4 and progression of hepatocellular carcinoma. J Surg Oncol 107: 105-110, 2013.

17. Bartley AN, Yao H, Barkoh BA, Ivan C, Mishra BM, Rashid A, Calin GA, Luthra R and Hamilton SR: Complex patterns of altered MicroRNA expression during the adenoma-adenocarcinoma sequence for microsatellite-stable colorectal cancer. Clin Cancer Res 17: 7283-7293, 2011.
18. Peres TV, Pedro DZ, de Cordova FM, Lopes MW, Goncalves FM, Mendes-de-Aguiar CB, Walz R, Farina M, Aschner M and Leal RB: In vitro manganese exposure disrupts MAPK signaling pathways in striatal and hippocampal slices from immature rats. Biomed Res Int 2013: 769295, 2013.

19. Thomas GM and Huganir RL: MAPK cascade signalling and synaptic plasticity. Nat Rev Neurosci 5: 173-183, 2004.

20. Cargnello $M$ and Roux PP: Activation and function of the MAPKs and their substrates, the MAPK-activated protein kinases. Microbiol Mol Biol Rev 75: 50-83, 2011.

21. Guo G, Yao W, Zhang Q and Bo Y: Oleanolic acid suppresses migration and invasion of malignant glioma cells by inactivating MAPK/ERK signaling pathway. PLoS One 8: e72079, 2013

22. Su N, Peng L, Xia B, Zhao Y, Xu A, Wang J, Wang X and Jiang B: Lyn is involved in CD24-induced ERK1/2 activation in colorectal cancer. Mol Cancer 11: 43, 2012.

23. Gout S, Morin C, Houle F and Huot J: Death receptor-3, a new E-Selectin counter-receptor that confers migration and survival advantages to colon carcinoma cells by triggering p38 and ERK MAPK activation. Cancer Res 66: 9117-9124, 2006.

24. Chen L, Li M, Li Q, Wang CJ and Xie SQ: DKK1 promotes hepatocellular carcinoma cell migration and invasion through $\beta$-catenin/MMP7 signaling pathway. Mol Cancer 12: 157, 2013.

25. Scanlon CS, Van Tubergen EA, Inglehart RC and D'Silva NJ: Biomarkers of epithelial-mesenchymal transition in squamous cell carcinoma. J Dent Res 92: 114-121, 2013

26. Parri M and Chiarugi P: Rac and Rho GTPases in cancer cell motility control. Cell Commun Signal 8: 23, 2010.

27. Yilmaz M and Christofori G: EMT, the cytoskeleton, and cancer cell invasion. Cancer Metast Rev 28: 15-33, 2009.

28. Slaby O, Svoboda M, Michalek J and Vyzula R: MicroRNAs in colorectal cancer: Translation of molecular biology into clinical application. Mol Cancer 8: 102, 2009.

29. Mraz M, Dolezalova D, Plevova K, Stano Kozubik K, Mayerova V, Cerna K, Musilova K, Tichy B, Pavlova S, Borsky M, et al: MicroRNA-650 expression is influenced by immunoglobulin gene rearrangement and affects the biology of chronic lymphocytic leukemia. Blood 119: 2110-2113, 2012.

30. Zuo ZH, Yu YP, Ding Y, Liu S, Martin A, Tseng G and Luo JH: Oncogenic activity of miR-650 in prostate cancer is mediated by suppression of CSR1 expression. Am J Pathol 185: 1991-1999, 2015.

31. Garkavtsev I, Kozin SV, Chernova O, Xu L, Winkler F, Brown E, Barnett GH and Jain RK: The candidate tumour suppressor protein ING4 regulates brain tumour growth and angiogenesis. Nature 428: 328-332, 2004.

32. Li X, Cai L, Liang M, Wang Y, Yang J and Zhao Y: ING4 induces cell growth inhibition in human lung adenocarcinoma A549 cells by means of Wnt-1/beta-catenin signaling pathway. Anat Rec (Hoboken) 291: 593-600, 2008.

33. Li S, Fan T, Liu H, Chen J, Qin C and Ren X: Tumor suppressor ING4 overexpression contributes to proliferation and invasion inhibition in gastric carcinoma by suppressing the NF-kappaB signaling pathway. Mol Biol Rep 40: 5723-5732, 2013.

34. Li J, Martinka M and Li G: Role of ING4 in human melanoma cell migration, invasion and patient survival. Carcinogenesis 29: 1373-1379, 2008

35. Hung T, Binda O, Champagne KS, Kuo AJ, Johnson K, Chang HY, Simon MD, Kutateladze TG and Gozani O: ING4 mediates crosstalk between histone $\mathrm{H} 3 \mathrm{~K} 4$ trimethylation and $\mathrm{H} 3$ acetylation to attenuate cellular transformation. Mol Cell 33: 248-256, 2009.

36. Lou C, Jiang S, Guo X and Dong XS: ING4 is negatively correlated with microvessel density in colon cancer. Tumour Biol 33: 2357-2364, 2012

This work is licensed under a Creative Commons Attribution-NonCommercial-NoDerivatives 4.0 International (CC BY-NC-ND 4.0) License. 